\title{
RESEARCH
}

Open Access

\section{MicroRNA-148a-3p inhibits progression of hepatocelluar carcimoma by repressing SMAD2 expression in an Ago2 dependent manner}

Zhao Huang ${ }^{1,2+}$, Jingyuan Wen ${ }^{1,2+}$, Jingjing $\mathrm{Yu}^{1,2}$, Jingyu Liao ${ }^{1,2}$, Sha Liu ${ }^{1,2}$, Ning Cai ${ }^{1,2}$, Huifang Liang ${ }^{1,2}$, Xiaoping Chen ${ }^{1,2,3,4,5^{*}}$, Zeyang Ding ${ }^{1,2,3,4,5^{*}}$ and Bixiang Zhang ${ }^{1,2,3,4,5^{*}}$

\begin{abstract}
Background: Hepatocellular carcinoma (HCC) is one of the most prevalent common cancer worldwide with high mortality. Transforming growth factor- $\beta$ (TGF- $\beta$ ) signaling pathway was reported dysregulated during liver cancer formation and progression. As a key component of TGF- $\beta$ signaling, the role of SMAD2 and its regulatory mechanisms in HCC remain unclear.

Methods: SMAD2 expression in paired HCC specimens were determined by western blot and immunohistochemistry (IHC). quantitative real-time PCR (qRT-PCR) was used to measure mRNA and microRNA (miRNA) expression level. Cell migration, invasion and proliferation ability were evaluated by transwell, CCK8 and EdU assay. In silico websites were used to manifest overall survival rates of HCC patients or to predict miRNAs targeting SMAD2. Dual luciferase reporter assay and anti-Ago2 immunoprecipitation assay were performed to confirm the binding between SMAD2 mRNA and miRNA-148a-3p (miR-148a). Tumorigenesis and lung metastasis mouse model were used to explore the role of miR-148a in vivo. In situ hybridization (ISH) was conducted to determine the expression of miR-148a in liver tissues.

(Continued on next page)
\end{abstract}

\footnotetext{
*Correspondence: chenxpchenxp@163.com; zyding@tjh.tjmu.edu.cn; bixiangzhang@163.com

'Zhao Huang and Jingyuan Wen contributed equally to this work. 'Hepatic Surgery Center, Tongji Hospital, Tongji Medical College, Huazhong University of Science and Technology, 1095 Jiefang Avenue, Wuhan 430030, China

Full list of author information is available at the end of the article
}

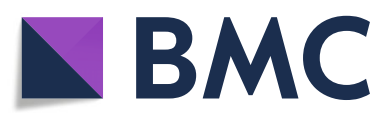

(- The Author(s). 2020 Open Access This article is licensed under a Creative Commons Attribution 4.0 International License, which permits use, sharing, adaptation, distribution and reproduction in any medium or format, as long as you give appropriate credit to the original author(s) and the source, provide a link to the Creative Commons licence, and indicate if changes were made. The images or other third party material in this article are included in the article's Creative Commons licence, unless indicated otherwise in a credit line to the material. If material is not included in the article's Creative Commons licence and your intended use is not permitted by statutory regulation or exceeds the permitted use, you will need to obtain permission directly from the copyright holder. To view a copy of this licence, visit http://creativecommons.org/licenses/by/4.0/. The Creative Commons Public Domain Dedication waiver (http://creativecommons.org/publicdomain/zero/1.0/) applies to the data made available in this article, unless otherwise stated in a credit line to the data. 


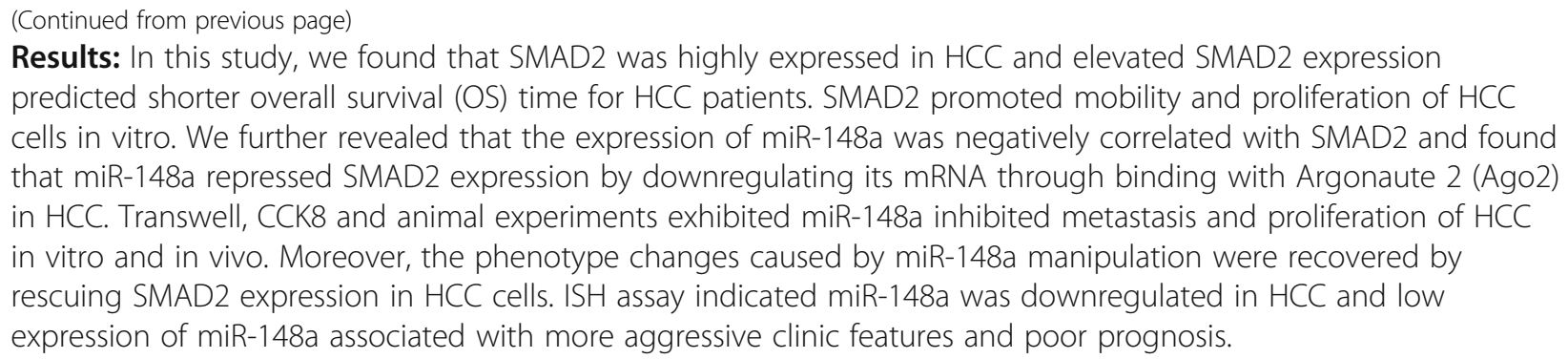

Conclusion: miR-148a was identified as a repressor of HCC progression by downregulating SMAD2 in an Ago2 dependent manner.

Keywords: HCC, SMAD2, miR-148a, Argonaute 2, TGF- $\beta$

\section{Background}

Liver cancer is the sixth most common cancer in incidence and the fourth leading cause of cancer-related death worldwide $[1,2]$. Hepatocellular carcinoma (HCC) is the major type of liver cancer, accounting for $75-85 \%$ of primary liver cancers [3]. The main risks for $\mathrm{HCC}$ are hepatitis $B$ virus (HBV), hepatitis $C$ virus $(\mathrm{HCV})$, aflatoxin contamination in food, alcohol abuse, obesity and so on [3]. Treatments for liver cancer patients contain surgical therapies, tumor ablation, transarterial therapies and systemic therapies [2]. However, the prognosis remains miserable, 5-years survival rate for liver cancer patients is $18 \%$, only inferior to pancreatic cancer [1].

TGF- $\beta$ signaling pathway has been reported playing a multifaceted role in tumor development and progression. It promotes homeostasis and suppresses tumor progression by inducing cytostasis, differentiation, apoptosis, inflammation depression and stroma-derived mitogens in normal and premalignant cells. Meanwhile, in advanced cancer cells, TGF- $\beta$ initiates immune evasion, growth factor production, differentiation to invasive phenotype, metastatic dissemination and metastatic colonies establishment [4]. Eight SMAD proteins were identified participating in TGF- $\beta$ signaling: the receptorregulated SMAD (R-SMAD) (SMAD1, 2, 3, 5 and 8), the co-mediator SMAD (Co-SMAD) (SMAD4), and the inhibitory SMAD (I-SMAD) (SMAD6 and 7) [5]. As RSMAD, SMAD2 and SMAD3 are phosphorylated by receptors of TGF- $\beta$ branch, whereas SMAD1, 5 and 8 are phosphorylated by other branches of receptors such as BMP receptors [6]. Upon TGF- $\beta$ stimulation, SMAD2 and SMAD3 are phosphorylated and activated by type I receptor kinase, and bind with SMAD4 to form activated SMAD complexes [5]. Regardless of the similar working mechanisms and high identity of protein structures [7], the role of SMAD2 and SMAD3 are often different, even opposite. Distinct effects of SMAD2 and SMAD3 were reported in breast cancer bone metastasis [8], pancreatic ductal adenocarcinoma cell proliferation and migration
[9], HaCaT keratinocyte cell growth [10], TGF- $\beta$ autoinduction in clostridium butyricum-activated dendritic cells [11] and TGF- $\beta$ induced transcription [12]. Our lab has previous reported SMAD3 promoted HCC metastasis by upregulating protein tyrosine phosphatase receptor epsilon (PTPRe) expression and high expression of SMAD3 was associated with poor prognosis in HCC [13], but the impacts of SMAD2 on HCC remain largely unclear.

MicroRNAs (miRNAs) are short endogenous RNAs 19-25 nucleotides in length that post-transcriptionally regulate mRNA expression [14]. Instead of directly silencing targeted mRNA, miRNAs in human and other bilaterian animals promote mRNA decay or repress mRNA translation mainly by recruiting RNA-induced silencing complex (RISC). As the heart of RISC, Argonaute2 (Ago2) recruits TNRC6, PABPC, and deadenylase complexes, i.e., the PAN2-PAN3 complex or CCR4-NOT complex to destabilize or repress mRNA [15-17]. Numerous miRNAs have found dysregulated in HCC and involved in HCC diagnosis, prognosis and therapeutics, such as, miR-16, miR-92a and miR-500 [18-20]. Lower miR-148a expression in HCC tissues was observed than that in adjacent noncancerous hepatic tissues and miR148a-3p expression was correlated to clinical TNM stage, metastasis, status of capsular infiltration and numbers of tumor nodes in HCC [21]. Xu et al. reported that hepatitis $B$ virus $\mathrm{X}$ protein $(\mathrm{HBx})$ repressed miR-148a expression and further upregulated the expression of hematopoietic pre-B cell leukemia transcription factor interacting protein (HPIP) to enhance tumorigenesis [22]. Gailhouste et al. revealed that miR-148a promoted hepatospecific phenotype of mouse fetal hepatoblasts (MFHs) and suppressed the invasiveness of transformed cells [23].

Our study revealed that SMAD2 exhibited oncogenic role in HCC and found that miR-148a was an upstream regulator of SMAD2 by decaying SMAD2 mRNA in an Ago2 dependent manner. miR-148a inhibited the 
mobility and proliferation of HCC cells and low expression of miR-148a in HCC was associated with shorter overall survival time. These results indicated miR-148a could be a promising diagnostic marker and therapeutic target for HCC patients.

\section{Methods}

\section{Patients and tissue specimens}

Human tumor and adjacent non-tumor tissues were collected from HCC patients underwent hepatectomy at the Hepatic Surgery Center, Tongji Hospital of Huazhong University of Science and Technology (HUST) (Wuhan, China). All procedures were approved by the Ethics Committee of Tongji Hospital, HUST and conducted according to the Declaration of Helsinki Principles. Prior written and informed consent was obtained from each patient.

\section{Cell lines and culture}

HCC cell lines MHCC-97H, HCC-LM3 were obtained from Liver Cancer Institute, Zhongshan Hospital, Fudan University, Shanghai, China. Hep3B, Huh7, PLC/PRF-5 (ALEX), HLF, Bel7402 and normal liver cell line HL7702 were purchased from China Center for Type Culture Collection (CCTCC, Wuhan, China). ALEX was purchased from cell bank of Chinese Academy of Sciences (Shanghai, China) [24]. All cell lines were maintained in Dulbecco Modified Eagle Medium (DMEM) (Hyclone, UT, USA) supplemented with $10 \%$ fetal bovine serum (FBS) (Gibico) at $37^{\circ} \mathrm{C}$ in a $5 \% \mathrm{CO}_{2}$ cell incubator.

\section{Cell counting kit 8 assay and EdU incorporation assay}

Cell Counting Kit 8 assay (Dojindo, Kumamoto, Japan) was performed according to manufactures' protocol. Briefly, 1500 indicated cells were seeded into 96-well plates. Culture medium was changed to $100 \mu \mathrm{l} 10 \%$ CCK8 solution at the indicated time and incubated in cell incubator for $2 \mathrm{~h}$. Optical density (O.D.) was measure by Universal Microplate Reader ELx 800 (BIO-TEK, USA) at $450 \mathrm{~nm}$ wave length. For each group, the absorbance values were measured by five replicates. After extracting blank value, an average of gross O.D. values was used for data analysis.

HCC cells (4000 cells/ well) were seeded into 96-well plates and cultured overnight for EdU incorporation assay by using Cell-Light ${ }^{\mathrm{tm}}$ EdU Apollo567 In Vitro Imaging Kit (Ribobio, Guangzhou, China) according to the manufacturer's instructions. Briefly, $100 \mu \mathrm{l} 50 \mu \mathrm{M}$ EdU solutions were added into cells and cultured for $2 \mathrm{~h}$. Cells were rinsed with PBS, fixed with $4 \%$ paraformaldehyde and incubated with $0.5 \%$ TritonX-100. Then, cells were stained with $100 \mu \mathrm{l} 1 \mathrm{X}$ Apollo solution for $30 \mathrm{~min}$, nucleus were stained with 1X Hoechst33342 solution. Representative images were captured with EVOS FL auto imaging system (life technologies, USA) and positive cells were counted by Image Pro. Plus version 6.0.

\section{Dual luciferase assay}

The sequence of 8284-8390, containing predicted binding site with miR-148a, in the 3 '-untranslated region (3'UTR) of SMAD2 or 3'UTR-SMAD2-mutant was cloned into the psiCHECK ${ }^{\mathrm{m}}$-2-vector (Promega, Madison, WI, United States). About $1 \times 10^{5}$ cells/well were seeded into 24-well plates. After $24 \mathrm{~h}$, the recombinant plasmid pSicheck-2-3'UTR-SMAD2 or pSicheck2-3'UTR-SMAD2-mutant were co-transfected into cells with miR-148a mimic, miR-148a inhibitor or their respective negative control (nc) using Lipofectamine 3000 (Invitrogen). Forty-eight h later, total protein from cells were extracted by Passive Lysis Buffer (Promega) and the luciferase activity was determined using DualLuciferase $^{\bullet}$ Reporter 1000 Assay System (Promega) by GloMax 20/20. Firefly luciferase values were normalized against Renilla luciferase activity, and the ratio of firefly/ Renilla luciferase activity was presented.

\section{Anti-Ago2 RNA binding protein immuno-precipitation (RIP) assay}

Rabbit anti-Ago2 IgG was purchased from Abcam (ab32381). Magna RIP ${ }^{\mathrm{m}}$ RNA-Binding Protein Immunoprecipitation Kit (Millipore, Darmstadt, Germany) was used to enrich Ago2 binding RNA. The enriched RNA was subjected to $\mathrm{qRT}$-PCR. $2^{-\Delta \mathrm{CT}}$ was calculated and normalized to the $2^{-\Delta \mathrm{CT}}$ of $10 \%$ input.

\section{Animal experiments}

4-5 weeks old male BALB/cA-nude mice were purchased from Beijing HFK Bioscience Co. Ltd. and maintained at SPF conditions. All animal experiments were approved by the Ethics Committee of Tongji Hospital, HUST. The whole procedure was in accordance with the "Guide for the Care and Use of Laboratory Animals" (NIH publication 86-23 revised 1985). For xenograft tumor model, $1 \times 10^{6}$ indicated tumor cells were suspended in $100 \mu \mathrm{l}$ serum free DMEM and inoculated subcutaneously into the flanks of nude mice. Thirty days later, all mice were sacrificed to compare the volume and weight of tumors. For lung metastasis model, $1 \times$ $10^{6}$ cells were suspended in $100 \mu \mathrm{l}$ serum free DMEM. Then cells were injected via the tail vein of nude mice. After 2 months of injection, mice were sacrificed and lungs were resected to calculate metastatic nodules.

\section{In situ hybridization (ISH) and immunohistochemistry analysis (IHC)}

ISH was performed using the ISH Kit (Boster, BioEngineering Company, Wuhan, China). All procedures followed the manufacturer's instructions. Samples were 
stained with hematoxylin, dehydrated with alcohol, washed with xylene, sealed with flavor sealing tablets. Oligo (5'Digoxin-ACAAAGTTCTGTAGTGCACTGA) was used as ISH probe for miR-148a. IHC staining was performed as described previously [24]. Primary antibody for SMAD2 (1:100, 12,570-1-AP) and Ki67 (1:100, 27,309-1-AP) were purchased from Proteintech. The representative images of ISH and IHC were captured and processed using DM2300 microscope and ScopeImage 9.0 software (Nanjing Jiangnan Novel Optics Co., Ltd., China). ISH and IHC staining scores were independently determined by 3 pathologists without prior knowledge of patient information. The overall score defined by multiplying the percentage of positive cells by the staining intensity score as described previously [24].

\section{In silico analysis and prediction websites}

Overall survival rates of $\mathrm{HCC}$ patients with different SMAD2 or SMAD3 level were analyzed by GEPIA [25] and Kaplan Meier-plotter [26] websites. TargetScan [27], miRTarBase [28] and miRcode [29] websites were used to predicted miRNAs targeting SMAD2 mRNA. StarBase [30] website was used to analyzed correlations between expression of miRNAs and SMAD2 mRNA, or overall survival rate of liver hepatocellular carcinoma (LIHC) patients with different miRNAs expression.

\section{Statistical analyses}

We used Prism 7.0 (GraphPad Software, La Jolla, CA, USA) or SPSS 13.0 (SPSS, Chicago, IL, USA) to analyze the data. Quantitative data were analyzed by Student's $t$ test (unpaired two-tailed comparison) or Pearson's correlation test. Kaplan-Meier and log-rank analysis were applied to evaluate survival between two groups. Categorical data were analyzed by chi-square test. All values were presented as mean \pm SEM. $P$ values less than 0.05 were considered statistically significant.

Supplementary materials and methods were provided in additional information.

\section{Result}

SMAD2 and SMAD3 are upregulated in HCC specimens and their high expression predict poor prognosis

We firstly examined the expression of SMAD2 and SMAD3 in 75 pairs of HCC and adjacent non-cancerous tissues by western blot (Fig. 1a and Supplementary Figure 1). The average band intensities of SMAD2 and SMAD3 were significantly higher in HCC tissues than adjacent non-cancerous tissues (Fig. $1 \mathrm{~b}$ and Supplementary Figure 2A) and their expression levels were correlated positively in HCC samples (Supplementary Figure 2B). We then explored the relevance between SMAD2 or SMAD3 expression and patient prognosis using GEPIA and Kaplan Meier-plotter websites, higher expression of SMAD2 predicted shorter overall survival (OS) in both patient cohort (Fig. 1c and d). While elevated expression of SMAD3 was associated with poorer prognosis only in GEPIA patient cohort (Supplementary Figure $2 \mathrm{C}$ and 2D). Given the role of SMAD3 in HCC had been investigated in detail [13], we focused on exploring the impacts of SMAD2 in HCC.

\section{SMAD2 promotes migration, invasion and proliferation of HCC cells}

The endogenous SMAD2 expression was found relatively low in Hep3B, ALEX, HLF and Bel7402 cells, meanwhile high in Huh7, MHCC-97H and HCC-LM3 cells (Fig. 2a). We successfully knocked down SMAD2 in MHCC-97H and Huh7 cells by two independent shRNA (MHCC-97H/sh SMAD2, Huh/sh SMAD2), and overexpressed SMAD2 in HLF and Hep3B cells (HLF/SMAD2, Hep3B/SMAD2) (Fig. 2b and Supplementary Figure 3A). Transwell assay indicated that overexpression of SMAD2 promoted and knockdown of SMAD2 inhibited migration and invasion in HCC cells (Fig. 2c and Supplementary Figure 3B). CCK8 and EdU assays showed that HCC cells with higher SMAD2 expression exhibited stronger proliferation ability compared with counterpart lower SMAD2 expression cells (Fig. 2d, e, Supplementary Figure $3 \mathrm{C}$ and $3 \mathrm{D})$.

\section{The mRNA of SMAD2 is targeted by miR-148a}

To identify the candidate upstream miRNAs of SMAD2, we used in silico websites to predict miRNAs which potentially bind with SMAD2 mRNA. Eleven miRNAs were co-predicted in TargetScan, miRTarBase and miRcode websites (Fig. 3a and Supplementary Table 1). Then, we overexpressed these 11 miRNAs respectively in MHCC97H and Huh7 cells by miRNA mimics, qRT-PCR revealed that SMAD2 mRNA level was downregulated after transfected with these miRNA mimics (Fig. 3b). Given miRNA mainly exerts its role by binding and decaying targeted mRNA [14, 17], we analyzed the mRNA expression relevance between SMAD2 and these miRNA in HCC samples by StarBase website. The mRNA level of miR-27b-3p (miR-27b) and miR-148a were found in negative correlation with SMAD2 mRNA (Fig. 3c, Supplementary Figure 4). We further analyzed the relevance between these 2 miRNAs and HCC patients' OS. Patients with higher miR-148a expression exhibited longer OS, while miR-27b showed no significant difference (Fig. 3d). Therefore, we speculated miR-148a was a potential regulator of SMAD2 in HCC.

\section{MiR-148a inhibits the expression of SMAD2 by downregulating its mRNA}

We stably overexpressed miR-148a in MHCC-97H and Huh7 cells (MHCC-97H/miR-148a, Huh7/miR-148a) 


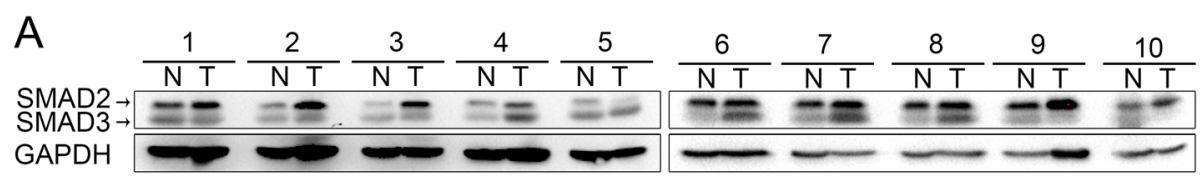

B

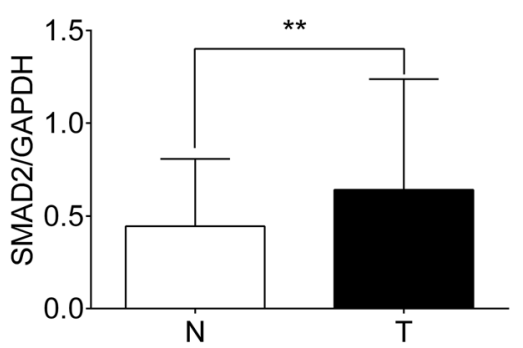

C

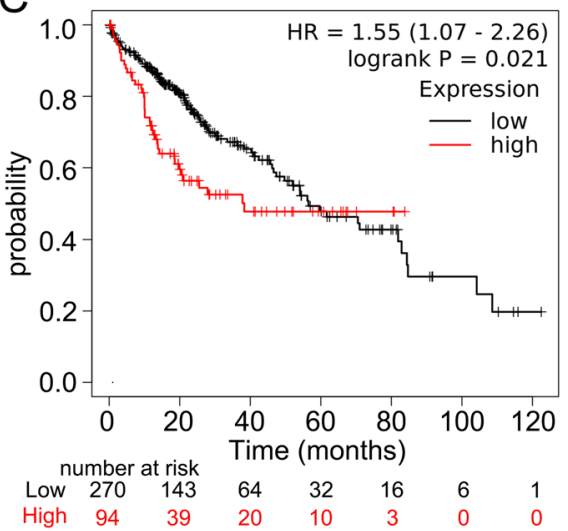

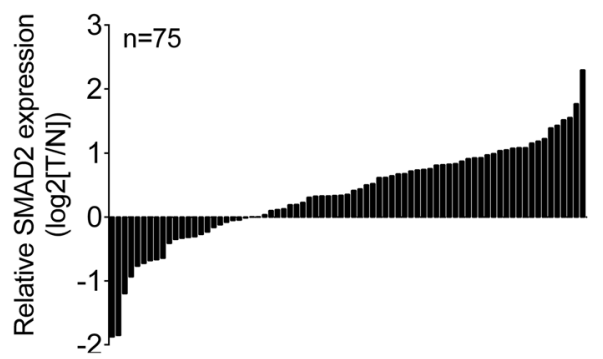

D

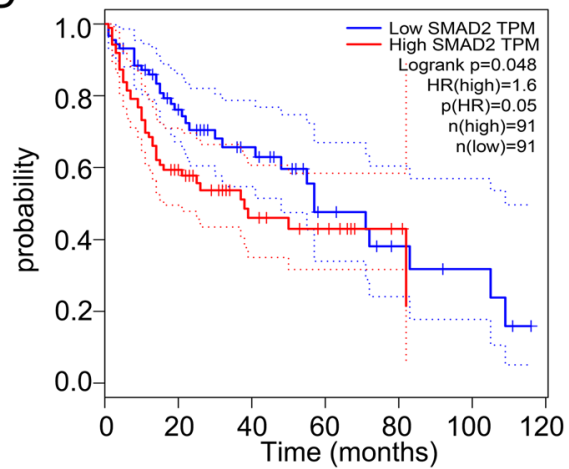

Fig. 1 SMAD2 is upregulated in HCC specimens and high expression of SMAD2 predicted poor prognosis. a Representative western blot bands of SMAD2 and SMAD3 in 75 pairs HCC clinic samples. GAPDH as loading control. $\mathbf{b}$ Quantification of SMAD2 bands intensity in liver tumor and non-tumorous tissues (left panel). Relative SMAD2 expression in HCC tissues by normalizing to their respective adjacent non-cancerous liver tissues (right panel). N, non-tumorous tissues. T, tumor tissues. c and $\mathbf{d}$ Kaplan-Meier analysis of the correlation between SMAD2 expression and overall survival in HCC patient cohorts by Kaplan Meier-plotter (c) or GEPIA (d) website. Data are shown as Mean \pm SEM. ${ }^{* *}, p<0.01$

with high endogenous SMAD2 level and knocked down miR-148a in HLF and Hep3B cells (HLF/KD miR-148a, Hep3B/KD miR-148a-) with low endogenous SMAD2 level by lentivirus (Fig. 4a). qRT-PCR and western blot demonstrated that overexpressing miR-148a downregulated and knocking down miR-148a upregulated the SMAD2 mRNA and protein level (Fig. 4b and c). TargetScan predicted that the position 8334-8340 of SMAD2 3' UTR was potential binding site of miR-148a. We constructed dual luciferase reporter plasmids pSicheck2-SMAD2-3'UTR and pSicheck2-SMAD2-3'UTR-mutant (Fig. 4d). Dual luciferase reporter assay showed that miR-148a mimic inhibited and miR-148a inhibitor enhanced reporter luciferase activity and the changes were abolished after mutating the binding site (Fig. 4e). Anti-Ago2 RNA immunoprecipitation (RIP) assays indicated that Ago2 enriched miR-148a and 3'UTR of SMAD2. The enrichments increased in miR-148a overexpressing MHCC-97H cells and decreased in miR148a knocking down HLF cells (Fig. 4f).
MiR-148a inhibits migration, invasion and proliferation ability of HCC cells in vitro

To investigated the role of miR-148a in HCC cells, we performed transwell and CCK8 assays after manipulating miR-148a expression. Overexpressing miR-148a inhibited migration and invasion in $\mathrm{MHCC}-97 \mathrm{H}$ and Huh7 cells, knocking down miR-148a promoted migration and invasion in HLF and Hep3B cells (Fig. 5a and Supplementary Figure 5A). CCK8 assay showed that overexpression of miR-148a inhibited and knockdown of miR-148a promoted proliferation in HCC cells (Fig. 5b and Supplementary Figure 5B).

\section{MiR-148a inhibits tumorigenesis and lung metastasis of HCC cells in vivo}

HCC cells were injected to nude mice subcutaneously to investigated the role of miR-148a on tumorigenesis. The volume and weight of subcutaneous tumors significantly decreased after overexpressing miR-148a in MHCC-97H 

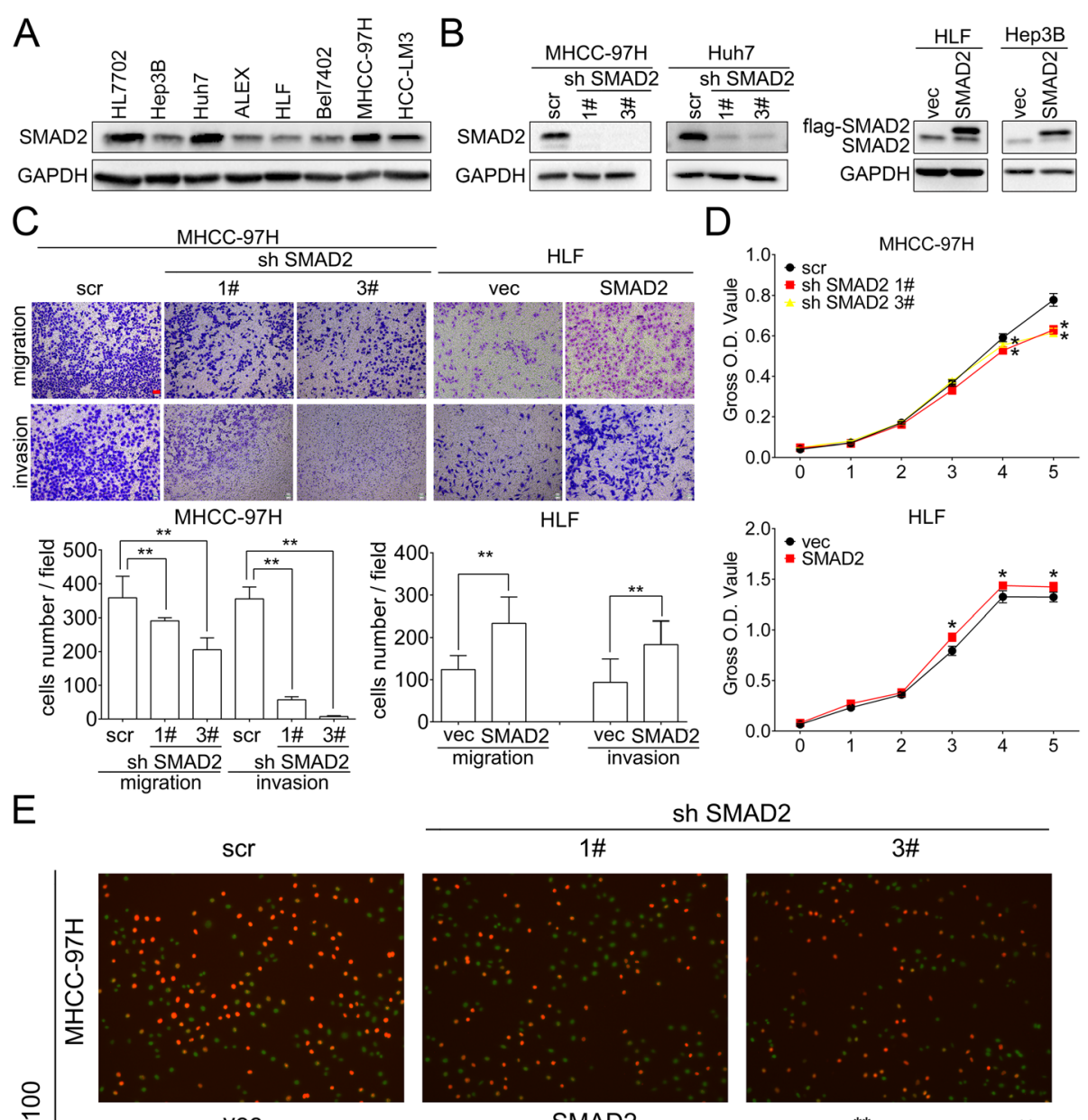

E
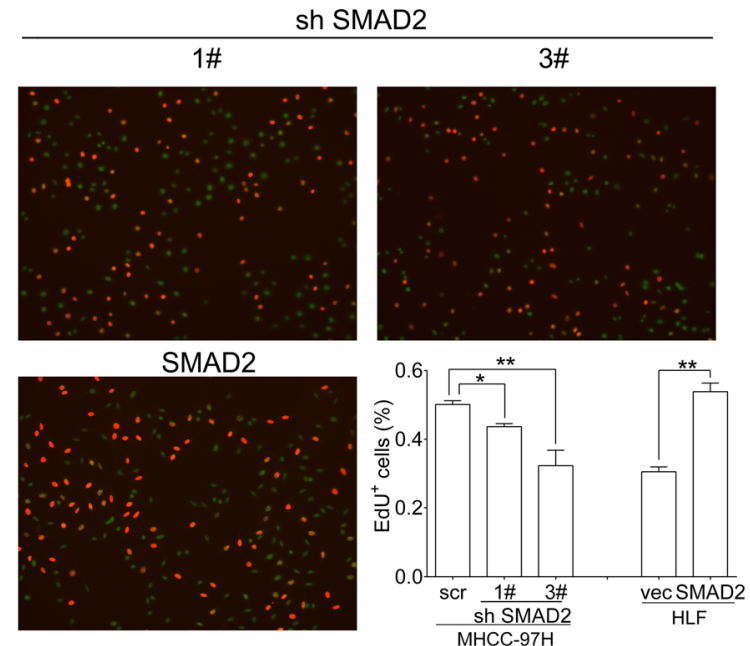

Fig. 2 SMAD2 promotes migration, invasion and proliferation of HCC cells. a Western blot analysis of endogenous SMAD2 expression in HCC cell lines. GAPDH as loading control. b Western blot analysis of SMAD2 knocking down or overexpression efficiency in the indicated cells. GAPDH as loading control. c Representative images of migration and invasion assays after stably knocking down or overexpressing SMAD2 in MHCC-97H or HLF cells (upper panel). Scale bar: red bar, $25 \mu \mathrm{m}$. Quantification of cells migrated or invaded (lower panel). d, e CCK8 and EdU assays after stably knocking down or overexpressing SMAD2 in MHCC-97H or HLF cells. Quantification of percentages of Edu ${ }^{+}$cells. Data are shown as Mean \pm SEM. *, $p<0.05 .{ }^{* *}, p<0.01$

cells and increased after knocking down miR-148a in HLF cells (Fig. 5c and Supplementary Figure 5C). IHC staining of SMAD2 and Ki67 indicated that HCC cells with higher miR-148a expression exhibited lower SMAD2 expression and weaker proliferation abilities (Fig. 5d). We then established HCC lung metastasis animal model by injecting cancer cells via tail veins. Less metastatic nodules were observed in post-mertem lungs of mice injected with miR-148a overexpressing MHCC-97H cells compared with control cells, and H\&E staining was further performed to verify the metastatic lesion formation. Similar results were obtained in mice injected with miR-148a knocking down HLF groups (Fig. 5e and Supplementary 5D).

The role of miR-148a in HCC cells was mediated by SMAD2 and low expression of miR-148a associated with more aggressive clinic features

We further overexpressed SMAD2 in MHCC-97H/miR148a, Huh7/miR-148a cells using pcDNA3.1-SMAD2 


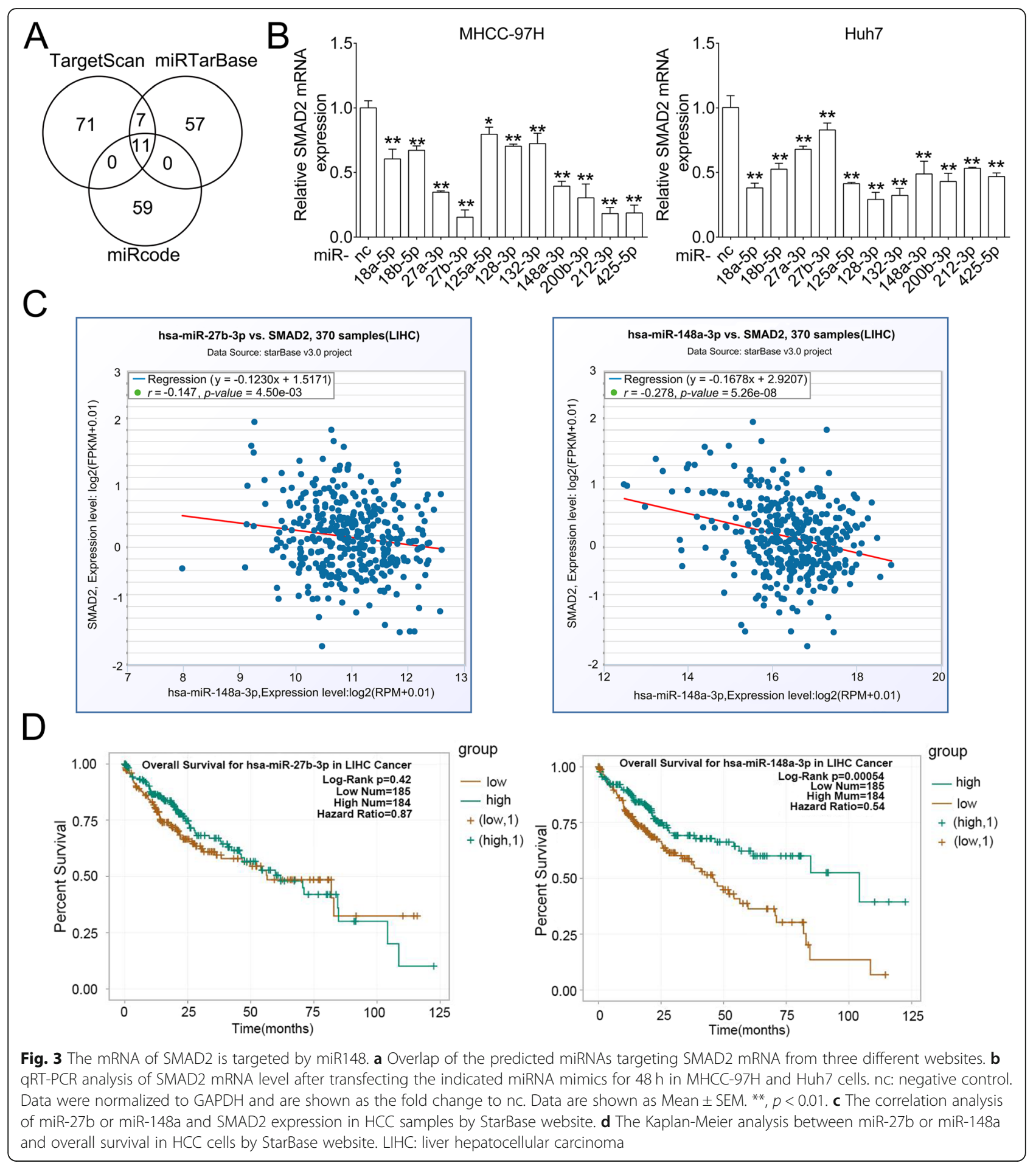

and stably knocked down SMAD2 in HLF/KD miR-148a, Hep3B/KD miR-148a cells (Fig. 6a and b). Transwell and CCK8 assay showed that the impaired migration, invasion and proliferation abilities in MHCC-97H/miR-148a, Huh7/miR-148a cells were recovered by overexpressing SMAD2. In contrast, the enhanced migration, invasion and proliferation abilities in HLF/KD miR-148a, Hep3B/
KD miR-148a cells were impeded by knockdown of SMAD2 (Fig. 6c-f).

ISH were performed in 77 pairs of HCC samples (Supplementary Table 2) to investigated the role of miR-148a in clinic. The average miR-148a staining score was significantly lower in HCC samples compared with adjacent non-cancerous tissues (Fig. 6g). 
A
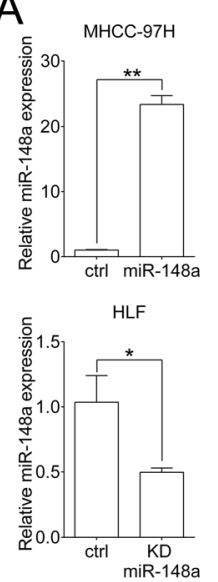

B $\mathrm{MHCC}-97 \mathrm{H}$
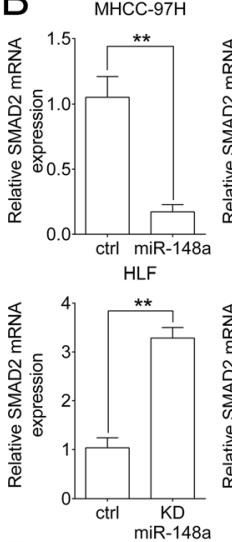

$\mathrm{F}$

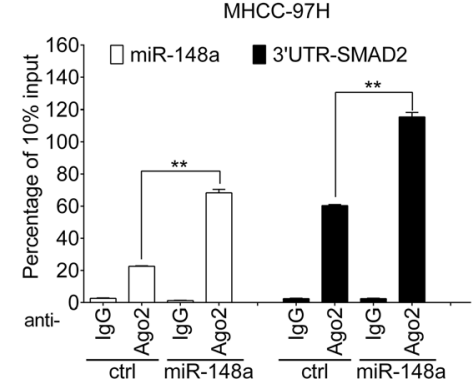

C
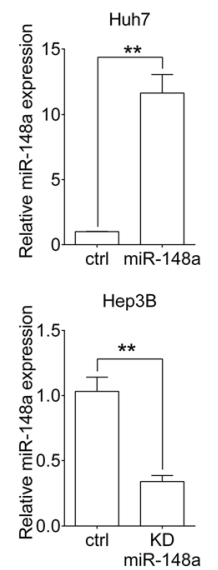

Huh7
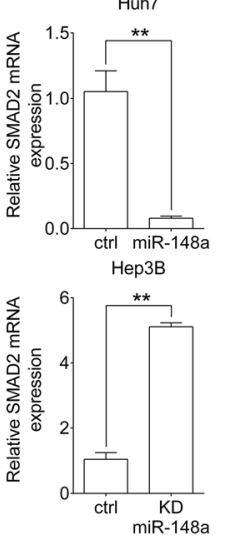

E

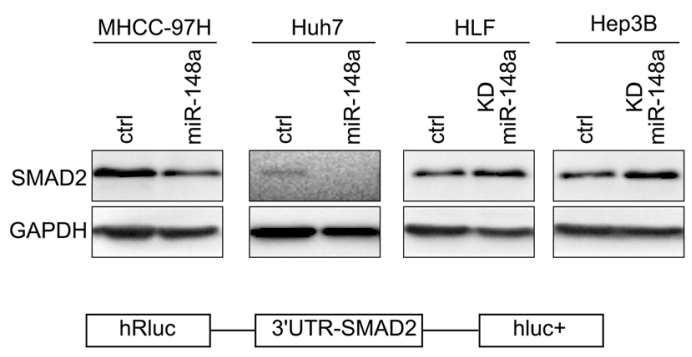

pSicheck2-SMAD2-3'UTR:

5' ...UGgCUGCAUCCUUGGUGCACUGU...3'

| | || | |

hsa-miR-148a: $\quad 3$ '...UGUUUCAAGACAUCACGUGACU...5'

pSicheck2-SMAD2-3'UTR-mutant:

5' ...UGgCugCAuCCuUgGaCGugACU...3'
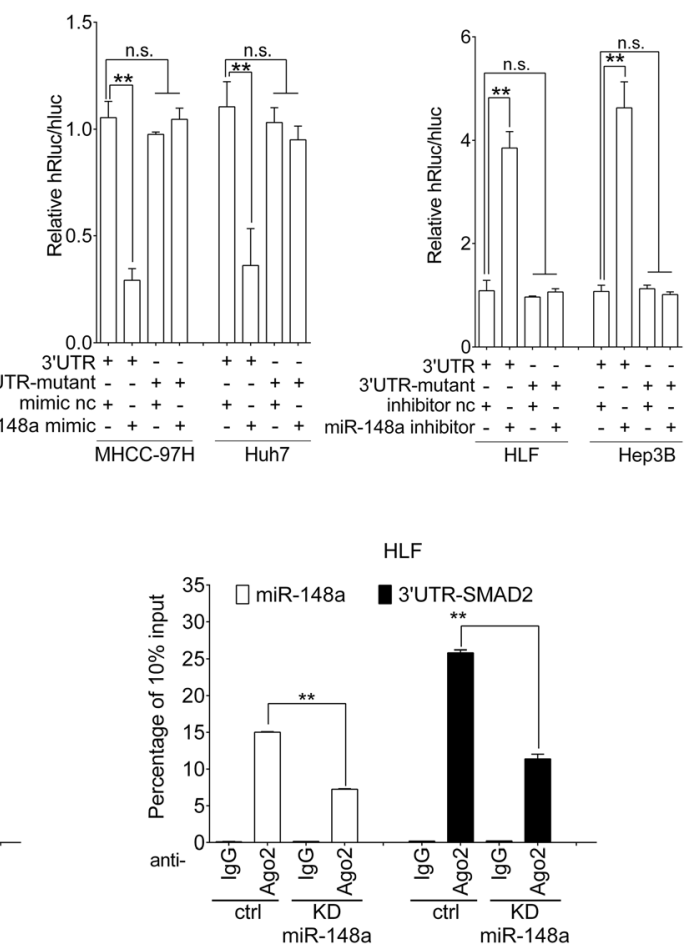

Fig. 4 miR-148a inhibits the expression of SMAD2 by downregulating its mRNA. a qRT-PCR analysis of miR-148a overexpression (miR-148a) or knocking down (KD miR-148a) efficacy in the indicated cells. Ctrl, cells transfected with control lentivirus. Data were normalized to U6 and are shown as the fold change to their respective control cells. $\mathbf{b}, \mathbf{c}$ qRT-PCR (b) and western blot (c) analysis of SMAD2 mRNA and protein level after stably overexpressing or knocking down miR-148a in MHCC-97H and Huh7 or HLF and Hep3B cells respectively. Data were normalized to GAPDH and are shown as the fold change to their respective control cells in (b). GAPDH, as loading control in (c). $\mathbf{d}$ Schematic image of binding site between miR-148a and SMAD2-3'-UTR. e The indicated cells were co-transfected with pSicheck2-SMAD2-3'UTR (pSicheck2-SMAD2-3'UTRmutant) and miR-148a mimic (miR-148a inhibitor). Forty-eight h after transfection, cells were subjected to dual luciferase assay. (f) RIP assays of miR-148a and 3'UTR-SMAD2 enrichments by anti-lgG or anti-Ago2 antibodies in miR-148a overexpressing MHCC-97H cells (left panel) and miR148a knocking down HLF cells (right panel). Data were normalized to $10 \%$ input. Data are shown as Mean \pm SEM. ${ }^{*}, p<0.05 .{ }^{* *}, p<0.01$. n.S., no significance

Chi-squared analysis indicated that lower expression of miR-148a was significantly associated with higher AFP level, HBV infection and higher Child-Pugh score (Supplementary Table 3).

\section{Discussion}

As R-SMAD proteins, the impacts of SMAD2 and SMAD3 on cancer initiation and progression were widely investigated. Interestingly, SMAD2 and SMAD3 

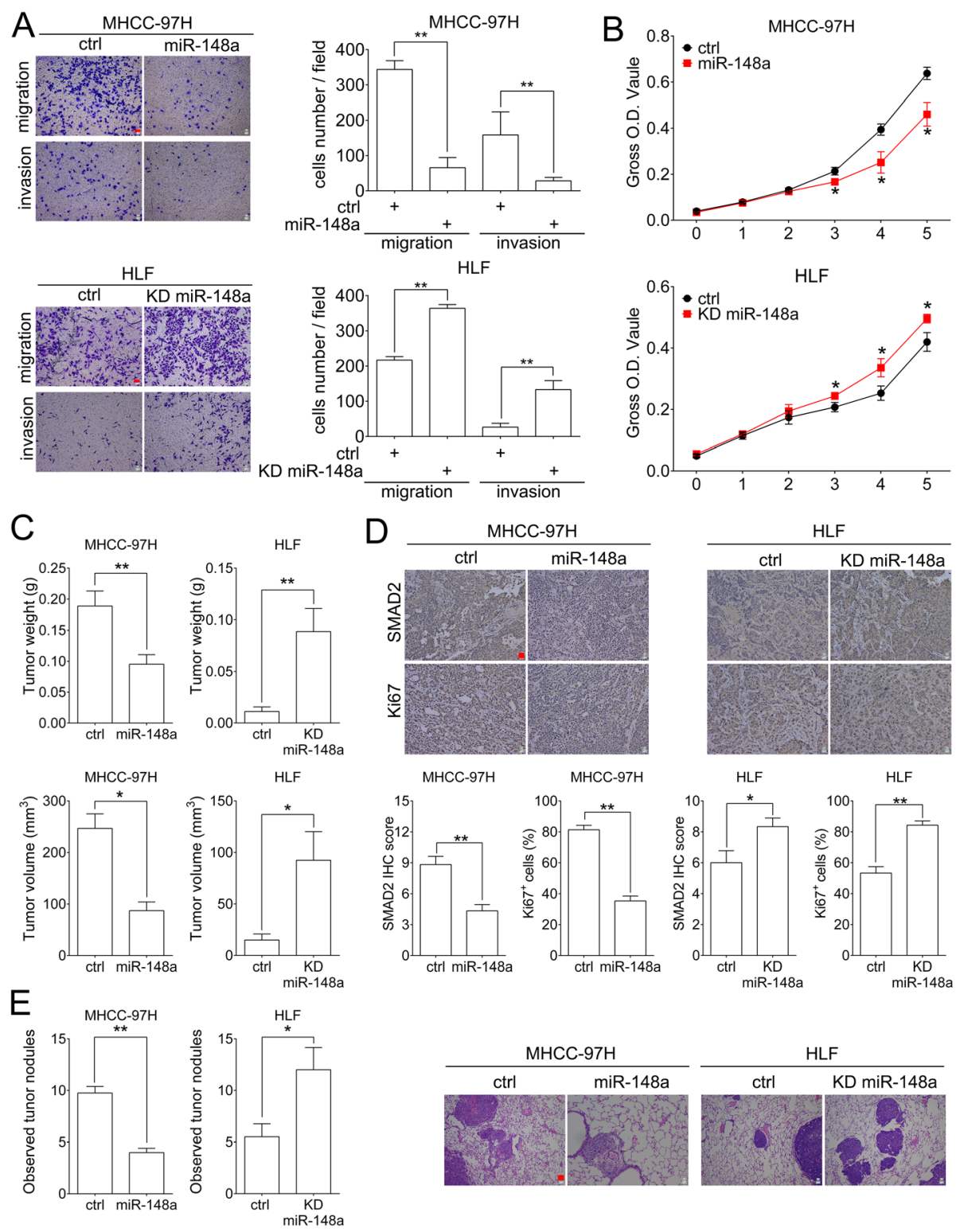

Fig. 5 miR-148a inhibits mobility and proliferation of HCC cells in vitro and in vivo. a Representative images of migration and invasion assay in the miR-148a overexpressing MHCC-97H or miR-148a knocking down HLF cells. Scale bar: red bar, $25 \mu \mathrm{m}$. Quantification of cells migrated and invaded. b CCK8 assay for the indicated cells. c BALB/c nude mice were subcutaneous injected with the indicated cell. Quantification for tumor volume and weight of subcutaneous tumors in the indicated groups. $\mathbf{d}$ Representative images of SMAD2 and Ki67 staining of subcutaneous tumors in the indicated groups. Scale bar: red bar, $25 \mu \mathrm{m}$. Quantification of SMAD2 IHC scores and Ki67 ${ }^{+}$HCC cell percentages. e HCC cells were injected into nude mice by tail veins to establish HCC lung metastasis animal model. Quantification of observed metastatic tumor nodules during autopsy and metastatic niches in H\&E stained slides. $\mathbf{f}$ Representative images of H\&E staining for lung. Scale bar: red bar, $25 \mu \mathrm{m}$. Data are shown as Mean \pm SEM. ${ }^{*}, p<0.05 .{ }^{* *}, p<0.01$

were found play distinct, even opposite roles under certain context [10-12]. In non-small-cell lung carcinoma (NSCLC), cancer metastasis was associated with inactivation of SMAD2-mediated and activation of SMAD3-mediated transcriptional programs [31]. During breast cancer bone metastasis progression, the TGF- $\beta$ induced bone metastatic genes expression were found depend on SMAD3 but not SMAD2, and knockdown of
SMAD3 in MDA-MB-231 cells inhibited bone metastasis, while SMAD3 knockdown led to a more aggressive phenotype [8]. In HCC, SMAD3 was reported suppressing carcinogenesis in chemically inducing animal models [32] and sustained SMAD3 activation promoted cancer metastasis [13]. However, the role of SMAD2 in HCC was poorly focused and remained obscure. Our results showed that the expression of SMAD2 was elevated in 

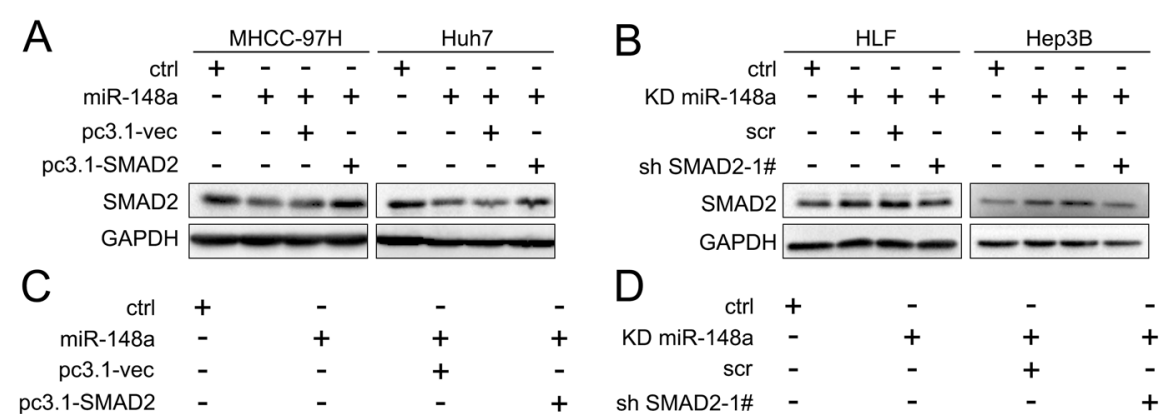

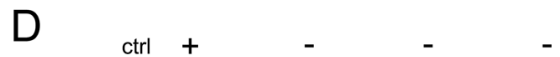
$\begin{array}{rllll}\mathrm{ctrl} & + & - & - & - \\ \mathrm{KD} \text { miR-148a } & - & + & + & + \\ \mathrm{scr} & - & - & + & - \\ \mathrm{sh} \text { SMAD2-1\# } & - & - & - & +\end{array}$
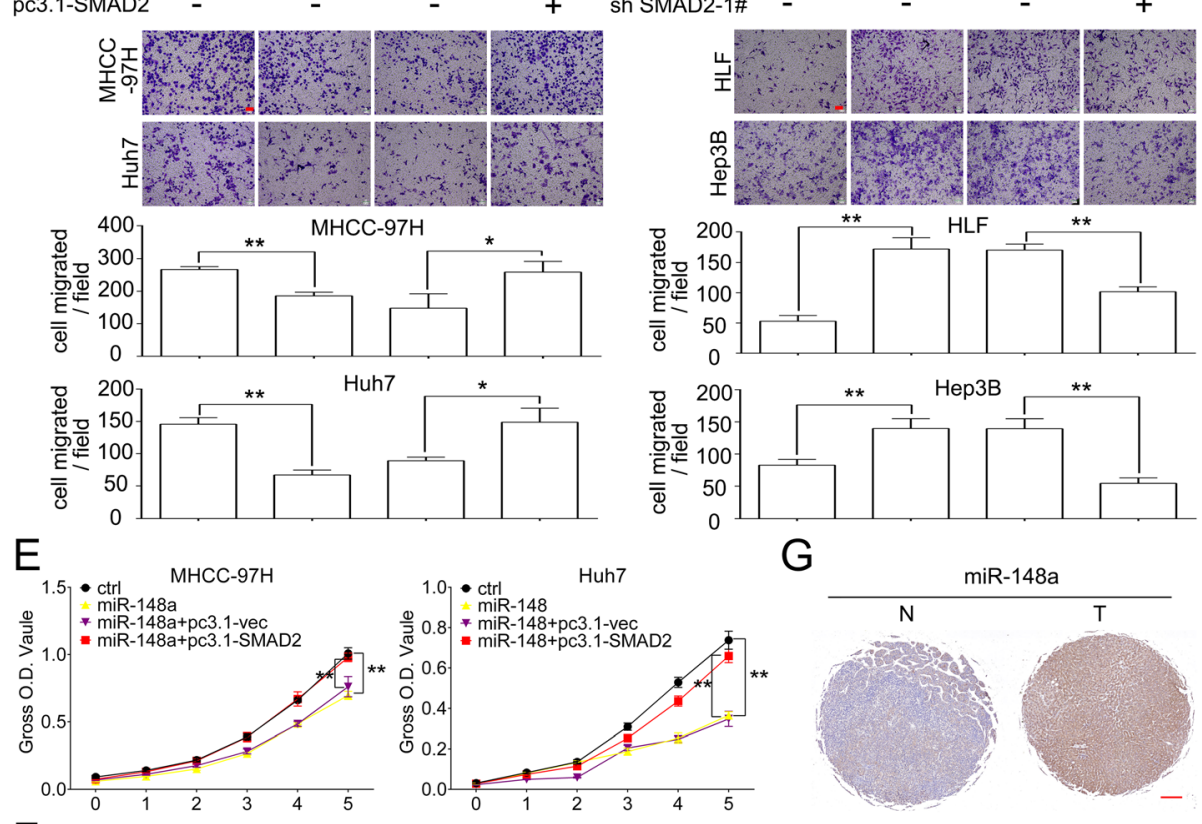

G

$\mathrm{F}$
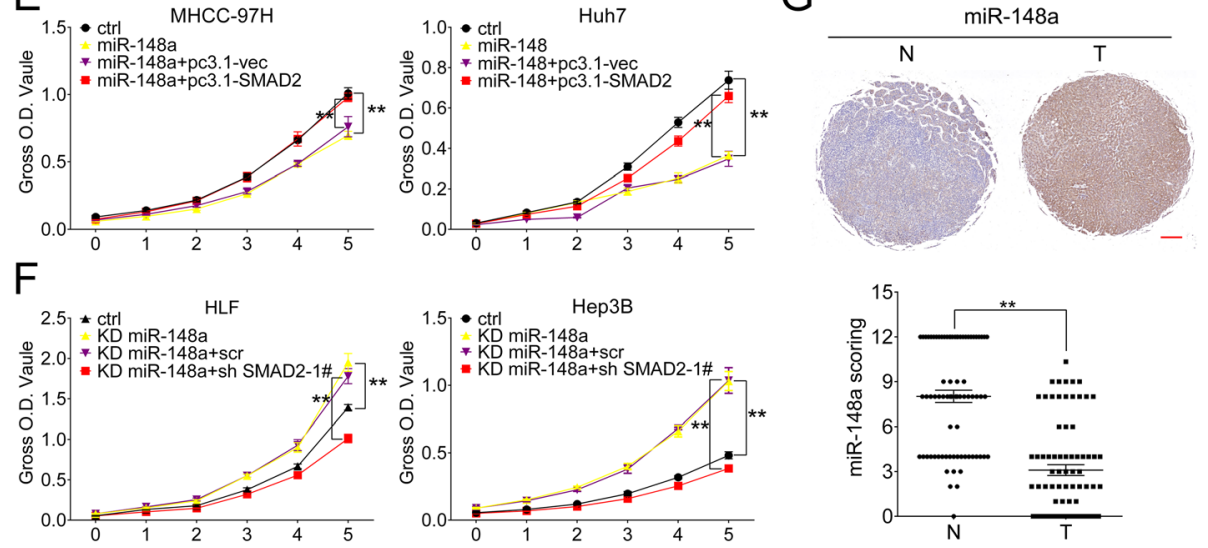

Fig. 6 The role of miR-148a in HCC cells was mediated by SMAD2. a pCDNA3.1-SMAD2 plasmids were transfected into miR-148a overexpressing MHCC-97H and Huh7 cells to transiently overexpress SMAD2 level. Forty-eight $h$ after transfection, cell lysates were subjected to western blot to evaluate SMAD2 expression. b SMAD2 were stably knocked down in miR-148a knocking down HLF and Hep3B cells. Western blot was performed to analyze SMAD2 knocking down efficacy. GAPDH as loading control in $(\mathbf{a}, \mathbf{b})$. $\mathbf{c}, \mathbf{d}$ Representative images of migration assay in the indicated cells (c, d, upper panel). Quantification of cells migrated (c, d lower panel). Scale bar, red bar, $25 \mu \mathrm{m}$. e, $\mathbf{f}$ CCK8 assay in the indicated cells. $\mathbf{g}$ Representative ISH images of miR-148a in paired HCC samples (upper panel). Scale bar, red, bar, $200 \mu \mathrm{m}$. ISH scoring of miR-148a in 77 pairs of HCC tissues (lower panel). N, non-cancerous tissues. T, tumor tissues. Data are shown as Mean \pm SEM. ${ }^{*}, p<0.05 .{ }^{*}, p<0.01$

HCC specimens and high expression of SMAD2 in HCC associated with poor prognosis. SMAD2 promoted proliferation, migration and invasion of HCC cells.

miRNAs can affect TGF- $\beta$ signaling process by directly targeting canonical members in the signaling pathway or targeting its effector genes [33]. In human, instead of directly slicing targeting mRNA by associated with an Ago protein that retained the catalytic ability of endonucleolytic cleavage, miRNAs mainly partially paired with targeted mRNA, and recruited RISC complexes to enhance mRNA decay or translational repression [17]. In our study, we predicted the mRNA of SMAD2 can be targeted by serials of miRNAs. Among these miRNAs, we identified miR-148a as an upstream regulator of SMAD2 by analyzing the expression relevance between miRNAs and SMAD2, and the association between miRNAs expression and OS in HCC. miR-148a was found downregulated in HCC tissue and low expression of miR-148a was associated with shorter OS time, which is consistent with previous study [21]. We further found 
that miR-148a downregulating SMAD2 expression through binding with Ago2, the core protein of RISC complexes.

Endeavors have been paid on exploring the effects of miR-148a on the HCC formation and progression. Researchers revealed that miR-148a promoted mouse fetal hepatoblasts (MFHs) differentiating to mature hepatocytes by directly targeting DNA methyltransferase 1 (DNMT1) [23] and induced hepatocytic differentiation to $\mathrm{HCC}$ by inhibiting IKK $\alpha / \mathrm{NUMB} / \mathrm{NOTCH}$ signaling [34]. While, Xiaojie Xu et al. found that Hepatitis B virus $\mathrm{X}$ protein $(\mathrm{HBx})$ repressed miRNA-148a to enhance tumorigenesis in mouse animal model of HCC [22]. The role of miRNA-148a as an HCC metastasis repressor seems consistent across different studies, miR-148a-3p suppressed the invasiveness of $\mathrm{HCC}$ cell by regulating cMet [23], Wnt1 [35] or activin A receptor type 1 (ACVR1) [36]. Our results showed that miR-148a inhibited proliferation and migration of $\mathrm{HCC}$ cells in vivo and in vitro. And the impacts of miR-148a in HCC cells were mediated by SMAD2.

\section{Conclusions}

This study demonstrated that SMAD2 was highly expressed in HCC specimens, elevated SMAD2 expression was associated with shorter overall survival time for HCC patients. SMAD2 played its tumor promoter role by enhancing migration, invasion and proliferation abilities of HCC cells. Besides, the expression of miR-148a was found negatively related with SMAD2 in HCC. Low expression of miR-148a was associated with more aggressive clinical features and predicted poorer prognosis. miR-148a inhibited HCC cells migration, invasion and proliferation in vitro, suppress tumorigenesis and metastasis in vivo. In mechanism, miR-148a recruited Ago2, bound with and decayed SMAD2 mRNA to inhibit SMAD2 expression. In conclusion, miR-148a was identified as a regulator of oncogenic SMAD2 and may serve as a promising prognostic marker or therapeutic target for HCC patients.

\section{Supplementary information}

Supplementary information accompanies this paper at https://doi.org/10. 1186/s13046-020-01649-0.

Additional file 1: Supplementary Figure 1. The expression of SMAD2 and SMAD3 are elevated in HCC tissues compared with counterpart nontumorous tissues.

Additional file 2: Supplementary Figure 2. SMAD3 is upregulated in HCC tissues and high expression of SMAD3 predicted shorter overall survival time.

Additional file 3: Supplementary Figure 3. SMAD2 promotes migration, invasion and proliferation of HCC cells.

Additional file 4: Supplementary Figure 4. The correlation between the expression of the indicated miRNAs and SMAD2 in HCC patients.
Additional file 5: Supplementary Figure 5. miR-148a inhibits metastasis and proliferation of HCC cells in vitro and in vivo.

Additional file 6: Supplementary Table 1. The miRNAs co-predicted by three different websites. Supplementary Table 2. Clinicopathologic characteristics of patients with hepatocellular carcinoma. Supplementary Table 3. Correlation between relative has-miR-148a expression and clinicopathologic characteristics in HCC patients $(n=77)$.

\section{Abbreviations}

3'UTR: 3'-Untranslated region; Ago2: Argonaute 2; ACVR1: Activin A receptor type 1; CCK8: Cell counting kit 8; Co-SMAD: Co-mediator SMAD;

DNMT1: DNA methyltransferase 1; HBX: Hepatitis B virus X protein; HBV: Hepatitis B virus; HCC: Hepatocellular carcinoma; HCV: Hepatitis C virus; HPIP: Hematopoietic pre-B cell leukemia transcription factor interacting protein; IHC: Immunohistochemistry; ISH: In situ hybridization; I-

SMAD: Inhibitory SMAD; LIHC: Liver hepatocellular carcinoma; MFHs: Mouse fetal hepatoblasts, i miRNA: microRNA; miR-148a: miR-148a-3p; miR-27b: miR27b-3p; NSCLC: Non-small-cell lung carcinoma; OS: Overall survival;

PTPRE: Protein tyrosine phosphatase receptor epsilon; qRT-PCR: Quantitative real-time PCR; RIP: Immunoprecipitation; RISC: RNA-induced silencing complex; R-SMAD: Receptor-regulated SMAD; TGF- $\beta$ : Transforming growth factor- $\beta$

\section{Acknowledgements}

As the first author of this study, I (Zhao Huang) want to thank my wife and her mother for taking care of our baby when I was engaged in this manuscript and thank my friends for supporting me in my daily expense.

\section{Authors' contributions}

$\mathrm{ZH}$ : Study conception and draft the manuscript; ZH and JW: experiment design, data acquisition and interpretation, statistical analysis; JW, JY, JL and NC, data acquisition; SL and HL, technical or material support; XC, ZD and BZ: study supervision and obtained funding. All authors read and approved the final manuscript.

\section{Funding}

This research was supported by the National Natural Science Foundation of China (No.81372327, 81572427, 81874189, 81572855, 81874065 and 81874149), the Hepato-Biliary-Pancreatic Investigation Fund of Chen Xiaoping Foundation for the Development of Science and Technology of Hubei Province (CXPJJH1 1800001-2018356), the State Key Project on Infection Disease of China (No. 2018ZX10723204-003), the National Key Research and Development Program of China (2018YFA0208904), Major Technological Innovation Projects of Hubei Province (2018ACA137), the HCP project of Huazhong University of Science and Technology (No. 5001540006, 5001540059). The funders had no role in study design, data collection and analysis, decision to publish, or preparation of the manuscript.

\section{Availability of data and materials}

All data generated or analyzed during this study are included in this published article and its supplementary information files.

\section{Ethics approval and consent to participate}

All experiments containing patient specimens or data were approved by the Ethics Committee of Tongji Hospital, HUST and were performed in accordance with the Declaration of Helsinki Principles. Prior written and informed consent was obtained from each patient.

\section{Consent for publication}

All materials and images are original. No consent needs to declare.

\section{Competing interests}

The authors declare no competing interest.

\section{Author details}

'Hepatic Surgery Center, Tongji Hospital, Tongji Medical College, Huazhong University of Science and Technology, 1095 Jiefang Avenue, Wuhan 430030, China. ${ }^{2}$ Clinical Medical Research Center of Hepatic Surgery at Hubei Province, Wuhan, China. ${ }^{3}$ Key Laboratory of Organ Transplantation, Ministry of Education, Wuhan, China. ${ }^{4}$ Key Laboratory of Organ Transplantation, 
National Health Commission, Wuhan, China. ${ }^{5}$ Key Laboratory of Organ Transplantation, Chinese Academy of Medical Science, Wuhan, China.

Received: 13 May 2020 Accepted: 21 July 2020

Published online: 04 August 2020

\section{References}

1. Villanueva A. Hepatocellular carcinoma. N Engl J Med. 2019;380:1450-62.

2. Yang JD, Hainaut P, Gores GJ, Amadou A, Plymoth A, Roberts LR. A global view of hepatocellular carcinoma: trends, risk, prevention and management. Nat Rev Gastroenterol Hepatol. 2019;16:589-604.

3. Bray F, Ferlay J, Soerjomataram I, Siegel RL, Torre LA, Jemal A. Global cancer statistics 2018: GLOBOCAN estimates of incidence and mortality worldwide for 36 cancers in 185 countries. CA Cancer J Clin. 2018;68:394-424.

4. Massague J. TGFbeta in cancer. Cell. 2008;134:215-30.

5. Shi Y, Massague J. Mechanisms of TGF-beta signaling from cell membrane to the nucleus. Cell. 2003;113:685-700.

6. Massague J. A very private TGF-beta receptor embrace. Mol Cell. 2008;29: 149-50.

7. Macias MJ, Martin-Malpartida P, Massague J. Structural determinants of Smad function in TGF-beta signaling. Trends Biochem Sci. 2015;40:296-308.

8. Petersen M, Pardali $E$, van der Horst $G$, Cheung $H$, van den Hoogen C, van der Pluijm G, Ten Dijke P. Smad2 and Smad3 have opposing roles in breast cancer bone metastasis by differentially affecting tumor angiogenesis. Oncogene. 2010;29:1351-61.

9. Ungefroren $\mathrm{H}$, Groth $\mathrm{S}$, Sebens $\mathrm{S}$, Lehnert $\mathrm{H}$, Gieseler F, Fandrich F. Differential roles of Smad2 and Smad3 in the regulation of TGF-beta1mediated growth inhibition and cell migration in pancreatic ductal adenocarcinoma cells: control by Rac1. Mol Cancer. 2011;10:67.

10. Brown KA, Pietenpol JA, Moses HL. A tale of two proteins: differential roles and regulation of Smad 2 and Smad3 in TGF-beta signaling. J Cell Biochem. 2007;101:9-33

11. Kashiwagi I, Morita R, Schichita T, Komai K, Saeki K, Matsumoto M, Takeda K, et al. Smad2 and Smad3 inversely regulate TGF-beta autoinduction in Clostridium butyricum-activated dendritic cells. Immunity. 2015;43:65-79.

12. Labbe E, Silvestri C, Hoodless PA, Wrana JL, Attisano L. Smad2 and Smad3 positively and negatively regulate TGF beta-dependent transcription through the forkhead DNA-binding protein FAST2. Mol Cell. 1998:2:109-20

13. Liao Z, Chen L, Zhang X, Zhang H, Tan X, Dong K, Lu X, et al. PTPRepsilon acts as a metastatic promoter in hepatocellular carcinoma by facilitating recruitment of SMAD3 to TGF-beta receptor 1. Hepatology. 2020. https:// doi.org/10.1002/hep.31104

14. Lu TX, Rothenberg ME. MicroRNA. J Allergy Clin Immunol. 2018;141:1202-7.

15. Chipman LB, Pasquinelli AE. miRNA targeting: growing beyond the seed. Trends Genet. 2019;35:215-22.

16. Elkayam E, Kuhn CD, Tocilj A, Haase AD, Greene EM, Hannon GJ, Joshua-Tor $L$. The structure of human argonaute-2 in complex with miR-20a. Cell. 2012; 150:100-10.

17. Bartel DP. Metazoan MicroRNAs. Cell. 2018;173:20-51.

18. Yang N, Ekanem NR, Sakyi CA, Ray SD. Hepatocellular carcinoma and microRNA: new perspectives on therapeutics and diagnostics. Adv Drug Deliv Rev. 2015;81:62-74

19. Mott JL. MicroRNAs involved in tumor suppressor and oncogene pathways: implications for hepatobiliary neoplasia. Hepatology. 2009;50:630-7.

20. Giordano S, Columbano A. MicroRNAs: new tools for diagnosis, prognosis, and therapy in hepatocellular carcinoma? Hepatology. 2013;57:840-7.

21. Pan L, Huang S, He R, Rong M, Dang Y, Chen G. Decreased expression and clinical significance of miR-148a in hepatocellular carcinoma tissues. Eur J Med Res. 2014;19:68.

22. Xu X, Fan Z, Kang L, Han J, Jiang C, Zheng X, Zhu Z, et al. Hepatitis B virus X protein represses miRNA-148a to enhance tumorigenesis. J Clin Invest. 2013; 123:630-45.

23. Gailhouste L, Gomez-Santos L, Hagiwara K, Hatada I, Kitagawa N, Kawaharada K, Thirion M, et al. miR-148a plays a pivotal role in the liver by promoting the hepatospecific phenotype and suppressing the invasiveness of transformed cells. Hepatology. 2013;58:1153-65.

24. Ding $Z Y$, Jin GN, Wang W, Chen WX, Wu YH, Ai X, Chen $L$, et al. Reduced expression of transcriptional intermediary factor 1 gamma promotes metastasis and indicates poor prognosis of hepatocellular carcinoma. Hepatology. 2014;60:1620-36.
25. Tang Z, Li C, Kang B, Gao G, Li C, Zhang Z. GEPIA: a web server for cancer and normal gene expression profiling and interactive analyses. Nucleic Acids Res. 2017;45:W98-W102.

26. Menyhart O, Nagy A, Gyorffy B. Determining consistent prognostic biomarkers of overall survival and vascular invasion in hepatocellular carcinoma. R Soc Open Sci. 2018;5:181006.

27. Lewis BP, Burge CB, Bartel DP. Conserved seed pairing, often flanked by adenosines, indicates that thousands of human genes are microRNA targets. Cell. 2005;120:15-20.

28. Chou CH, Shrestha S, Yang CD, Chang NW, Lin YL, Liao KW, Huang WC, et al. miRTarBase update 2018: a resource for experimentally validated microRNA-target interactions. Nucleic Acids Res. 2018;46:D296-302.

29. Jeggari A, Marks DS, Larsson E. miRcode: a map of putative microRNA target sites in the long non-coding transcriptome. Bioinformatics. 2012;28:2062-3.

30. Li JH, Liu S, Zhou H, Qu LH, Yang JH. starBase v2.0: decoding miRNA-ceRNA, miRNA-ncRNA and protein-RNA interaction networks from large-scale CLIPSeq data. Nucleic Acids Res. 2014;42:D92-7.

31. Ying Z, Tian H, Li Y, Lian R, Li W, Wu S, Zhang HZ, et al. CCT6A suppresses SMAD2 and promotes prometastatic TGF-beta signaling. J Clin Invest. 2017; 127:1725-40.

32. Millet $\mathrm{C}$, Zhang YE. Roles of Smad3 in TGF-beta signaling during carcinogenesis. Crit Rev Eukaryot Gene Expr. 2007;17:281-93.

33. Butz H, Racz K, Hunyady L, Patocs A. Crosstalk between TGF-beta signaling and the microRNA machinery. Trends Pharmacol Sci. 2012:33:382-93.

34. Jung KH, Zhang J, Zhou C, Shen H, Gagea M, Rodriguez-Aguayo C, LopezBerestein $\mathrm{G}$, et al. Differentiation therapy for hepatocellular carcinoma: multifaceted effects of miR-148a on tumor growth and phenotype and liver fibrosis. Hepatology. 2016;63:864-79.

35. Yan H, Dong X, Zhong X, Ye J, Zhou Y, Yang X, Shen J, et al. Inhibitions of epithelial to mesenchymal transition and cancer stem cells-like properties are involved in miR-148a-mediated anti-metastasis of hepatocellular carcinoma. Mol Carcinog. 2014;53:960-9.

36. Li L, Liu Y, Guo Y, Liu B, Zhao Y, Li P, Song F, et al. Regulatory MiR-148aACVR1/BMP circuit defines a cancer stem cell-like aggressive subtype of hepatocellular carcinoma. Hepatology. 2015;61:574-84.

\section{Publisher's Note}

Springer Nature remains neutral with regard to jurisdictional claims in published maps and institutional affiliations.

Ready to submit your research? Choose BMC and benefit from:

- fast, convenient online submission

- thorough peer review by experienced researchers in your field

- rapid publication on acceptance

- support for research data, including large and complex data types

- gold Open Access which fosters wider collaboration and increased citations

- maximum visibility for your research: over $100 \mathrm{M}$ website views per year

At $\mathrm{BMC}$, research is always in progress.

Learn more biomedcentral.com/submission 